\title{
Focusing Particles by Induced Charge Electrokinetic Flow in a Microchannel
}

\author{
Yongxin Song ${ }^{1,3}$, Chengfa Wang ${ }^{1,3}$, Mengqi $\mathrm{Li}^{2}$, Xinxiang $\operatorname{Pan}^{1}$ and Dongqing $\mathrm{Li}^{* 2}$
}

1 Department of Marine Engineering, Dalian Maritime University, Dalian, 116026, China

2 Department of Mechanical and Mechatronics Engineering, University of Waterloo, Waterloo, ON, N2L 3G1, Canada

*Corresponding authors: E-mail: dongqing.li@uwaterloo.ca

\section{Statement}

This is the peer reviewed version of the following article: Song, Y., Wang, C., Li, M., Pan, X. and Li, D. (2016), Focusing particles by induced charge electrokinetic flow in a microchannel. ELECTROPHORESIS, 37: 666-675. doi:10.1002/elps.201500361, which has been published in final form at http://dx.doi.org/10.1002/elps.201500361. This article may be used for non-commercial purposes in accordance with Wiley Terms and Conditions for SelfArchiving.

Both authors contributed equally to this work. 


\begin{abstract}
A novel method of sheathless particle focusing by induced charge electrokinetic flow (ICEK) in a microchannel is presented in this paper. By placing a pair of metal plates on the opposite walls of the channel and applying an electrical field, particle focusing is achieved due to the two vortex pairs which constrain the flow of the particle solution. As an example, the trajectories of particles under different electrical fields with only one metal plate on one side channel wall were numerically simulated and experimentally validated. Other flow focusing effects, such as the focused width ratio (focused width/channel width) and length ratio (focused length/ half length of metal plate) of sample solution, were also numerically studied. The results show that the particle firstly will pass through the gaps between the upstream vortices and the channel walls. Afterwards, the particle will be focused to pass through the gap between the two downstream vortices which determine the focused particle position. Numerical simulations show that the focused particle stream becomes thin with the increase in the applied electrical field and the length of the metal plates. As regards to the focused length ratio of the focused stream, however, it slightly increases with the increase in the applied electrical field and almost keeps constant with the increase in the length of the metal plate. The size of the focused sample solution, therefore, can be easily adjusted by controlling the applied electrical field and the size of the metal plates.
\end{abstract}

Keywords: Particle focusing, Flow focusing, Induced charge electrokinetic flow, Microfluidic chip 


\section{Introduction}

Focusing particles or cells one by one to pass a detection region is often required for some biological processes [1], such as cell analysis with a flow cytometer [2], fluorescence detecting or sorting cells or particles [3]. For a conventional flow cytometer, two sheath streams, generated by mechanical pumps and flowing from the two sides of the sample stream, are generally used to focus the sample solution. For a portable microfluidic lab-on-chip device, however, new focusing techniques, other than using the bulky pumps and valves, have to be developed in order to minimize the device size.

Several methods have been proposed for particle focusing in microfluidic channels and can be generally categorized into sheath flow method and sheathless focusing approach. Sheath flow focusing, which employs one or two sheath fluids (boundary fluids) to squeeze the sample solution and thus focus the particles suspended in the sample solution, is the most popular focusing method for microfluidic devices. For this method, both 2D (two dimensions) and 3D (three dimensions) particle focusing can be achieved depending upon the number of sheath fluid. The width of the focused stream is mainly determined by the flow-rate ratio between the sheath and the sample streams [4]. Generally, in a microfluidic chip, the motion of the sheath fluid can be generated by either a pressure gradient or an electrical field. For the former type, it normally requires external pumps (e.g., syringe pumps), tubing and valves which are bulk in size and difficult to be integrated into the microfluidic device. As regards to the electrical-driven sheath flow (i.e., electroosmotic flow), a constant flow rate can be generated by applying a DC (Direct Current) potential difference across a channel [5-10]. The main advantage of using electroosmotic flow for particle focusing is no mechanical parts. However, a strong electric field is normally required which may destroy the cell membrane. The details about the related studies can be found in some review papers [1, 11-13].

To overcome the shortcomings of sheath flow focusing, especially the involvement of bulky external pumps, sheathless flow focusing methods are put forward. These methods work by manipulating the trajectories of particles by applying electrical, or acoustic, or magnetic [14], or optical [15] force. One typical electrical particle focusing technology is dielectrophoresis 
(DEP) which utilizes the interaction of the non-uniform electrical field and the particles [16]. The DEP force can be generated by both AC [17-24] (Alternative Current) and DC electrical fields [25]. Different focusing effects, such as 3D [26,27],'electrodeless' [28] and nanoparitcle focusing [29] have also been reported. The review on this technique can be found in the reference papers [30,31]. However, there are several disadvantages for this type of methods. For electrode-based AC dielectrophoresis, electrodes fabrication in microchannel generally requires complicated procedures and expensive equipment. For insulator based DC-DEP, high voltages, normally several hundred volts, have to be applied in order to generate sufficiently strong electrical fields. The strong electrical field can easily damage the biological sample, such as killing or lysing the cells.

Acoustophoretic based particle focusing, achieved by an acoustic-wave-induced radiation pressure on particle, has recently attracted large interest $[3,32-37]$ due to its relative simple operation. However, there are some integration challenges for this method. First, the transducers need to be placed closely on the sides of the focusing channel, which affects the integration with other functional components in a flow cytometer [38]. Furthermore, it normally requires a radio frequency (RF) signal generator and a power amplifier which are of bulky size. Inertial focusing, utilizing the inertia of the fluid to control particle's position, is another sheathless focusing method [39-43]. This method relies on a relative high flow rate in order to generate decent inertia motion and thus is suitable for high-speed applications. However, it is generally difficult to generate a high-speed flow in a microchannel. Moreover, the equilibrium positions of the focused particle streams are sensitive to the particle's Reynolds number [38].

As discussed above, while the sheath flow focusing is very efficient with little side effects, bulky off-chip devices are normally involved. The sheathless focusing method is more advantageous in integration with the microfluidic chip. However, how to make a balance between enlarging the manipulating force and minimizing the side effects at the same time is still challenging. In this paper, a novel sheathless particle focusing method by induced charge electrokinetic flow in a microchannel is presented. By placing two metal plates in a 
microchannel, one on one side wall, another on the opposite side wall, and applying an electrical field along the channel, the sample solution will be electrokinetically transported to downstream and squeezed by vortices generated over the metal plates. In this way, particle focusing is achieved without using sheath flow or any off-chip devices. While mixing enhancement with micro-votices generated by a time-dependent electrical field has been reported [44], there is not report on particle focusing with ICEO method.

\section{Working principle}

A schematic diagram of the proposed method is shown in Figure 1. The particle focusing system consists of a microfluidic chip with a straight channel and a DC power supply. Two metal plates are placed on two side channel walls facing each other respectively. There are an inlet well and an outlet well at the two ends of the microchannel. After adding the sample solution in the channel, a DC electrical field is applied via electrodes inserted in the two wells. The working principle of particle focusing can be explained as below:

As shown in Figure 1(a), when an electric field is applied across the channel from the left to the right, opposite charges are induced on the surface of the two metal plates. More specifically, negative charges will be induced on the left half of the metal surface, and positive charges will be induced on the right half of the metal surface. The surface charges will in turn attract the counter-ions in the liquid to form dipolar electrical double layers (EDL) along the metal surface (Figure 1 (a)).

Once the induced dipolar double layer is formed, the externally applied electrical field drives the ions and the liquid into motion and forms the well-known induced charge electrokinetic flow (ICEKF). The resultant electrokinetic flow will generate vortices near the metal surface due to opposite induced charges on the surface [45]. Due to the much stronger electroosmotic flow at metal surface, the two upstream vortexes act as two 'pumps' to transport the sample solution through the gap between the metal and vortex. The downstream vortices, behave like virtual 'columns' and force the solution to flow through the narrow passage between them, providing the potential for particle focusing (Figure 1 (b)). 
The flow velocity of the ICEKF decides the size of the vortex and thus the size of focused sample stream. Theoretically, the flow velocity $\left(U_{I C E K}\right)$ varies proportionally to the local tangential electric field $\mathrm{E}$ and is given by the Helmholtz-Smoluchowski equation [46]:

$$
U_{I C E K}=-\frac{\varepsilon_{w} \zeta_{i}}{\mu} E
$$

where $\varepsilon_{\mathrm{w}}$ and $\mu$ are the dielectric constant and the viscosity of the liquid; $\zeta_{i}$ is the induced zeta potential. Because of the dependence of the induced zeta potential $\left(\zeta_{i}\right)$ on the local electrical field, it is clear from Eq. (1) that the flow velocity (also the vortex size) can be regulated by varying the applied electrical field.

\section{Mathematical models}

\subsection{Electric field}

Once a voltage difference $(\Delta \psi)$ is exerted across the microchannel, the applied potential field through the system is governed by Laplace's equation [47]:

$$
\nabla^{2} \psi=0
$$

and the local applied electric field strength $(\vec{E})$ is given by:

$$
\vec{E}=-\nabla \psi
$$

The applied electric field will induce surface charges on the metal plates. The induced surface charges will in turn attract the counter-ions in the liquid to form electrical double layer. The induced zeta potential $\zeta_{i}$ is given by [45]:

$$
\zeta_{i}=-\psi+\frac{\int_{S} \psi d A}{\mathrm{~A}}
$$

where $S$ is the conducting metal surface in the applied electric field, A is the area of the entire surface of the metal plate.

The boundary conditions of the electric field are:

$$
\vec{n} \cdot \nabla \psi=0 \quad \text { at the channel walls and the particle surface }
$$


where $\vec{n}$ is the unit normal vector pointing from the boundary surface into the fluid.

$$
\begin{aligned}
& \psi=V_{0} \quad \text { at the channel inlet } \\
& \psi=0 \quad \text { at the channel outlet }
\end{aligned}
$$

\subsection{Flow field}

The flow field is governed by the continuity equation and the momentum equation:

$$
\begin{gathered}
\nabla \cdot \vec{U}=0 \\
\rho\left[\frac{\partial \vec{U}}{\partial t}+\vec{U} \cdot \nabla \vec{U}\right]=-\nabla p+\mu \nabla^{2} \vec{U}+\rho_{e} \vec{E}
\end{gathered}
$$

where $\rho$ is the density of the liquid, $\mu$ is the viscosity of the liquid, $\vec{U}$ is the velocity vector, $\nabla p$ is the pressure gradient, $\rho_{e}$ is the local net charge density.

In this study, no pressure gradient across the microchannel and the fluid flow is totally electrokinetically driven. The pressures at the two ends of the microchannel are set as zero:

$$
\mathrm{p}=0 \quad \text { at inlet and outlet }
$$

Also, the pressure gradient is set to be zero at the channel wall, considering no flux across the wall:

$$
\overrightarrow{\mathrm{n}} \cdot \nabla \mathrm{p}=0 \quad \text { at channel walls }
$$

Because the buffer solution is electric neutrality and the net charge density $\rho_{e}$ is non-zero only in the double layer, the body force $\rho_{e} \vec{E}$ is zero except in the double layer (EDL). In addition, the thickness of the EDL is considered to be very small (nanometer) which is negligible in comparison with the size of the microchannel. Therefore, there is no need to solve the flow field in the thin EDL. Instead, we use a slip velocity boundary for the flow field in the microchannel. According to the Helmholtz-Smoluchowski equation, the slip velocity boundary conditions are:

$$
\begin{array}{ll}
\vec{U}=-\frac{\varepsilon_{w} \zeta_{\mathrm{w}}}{\mu} \vec{E}, & \text { at the non-conducting channel wall } \\
\vec{U}=-\frac{\varepsilon_{w} \zeta_{i}}{\mu} \vec{E}, & \text { at the metal plate surface }
\end{array}
$$




$$
\vec{U}=\vec{U}_{p}+\vec{\omega}_{\mathrm{p}} \times\left(\vec{x}_{s}-\vec{x}_{p}\right)-\frac{\varepsilon_{w} \zeta_{p}}{\mu} \vec{E}, \quad \text { at particle surface }
$$

where $\zeta_{\mathrm{w}}$ is the zeta potential on the non-conducting channel wall, $\zeta_{\mathrm{i}}$ is the induced zeta potential on the metal plate surface as given by Eq. $(4), \zeta_{\mathrm{p}}$ is the zeta potential on the particle surface, $\vec{U}_{p}$ is the translational velocity of the particle, $\vec{\omega}_{\mathrm{p}}$ is the rotational velocity of the particle, $\vec{x}_{s}$ is the position vector on the particle surface, $\vec{x}_{p}$ is the position vector of the particle center.

For Eq.(11c), the first item represents the translocation velocity; the second item represents the rotation velocity and the third is the EOF flow of the solution.

\subsection{Particle motion}

The translational velocity of the particle is governed by Newton's second law,

$$
m_{p} \frac{d U_{p}}{d t}=\vec{F}_{n e t}
$$

where $m_{p}$ is the mass of the particle and $\vec{F}_{n e t}$ is the net force acting on the particle given by [48-50]:

$$
\vec{F}_{n e t}=\int \bar{\sigma}_{\mathrm{p}} \cdot \vec{n} d S
$$

where $S$ is the particle surface, $\bar{\sigma}_{\mathrm{p}}$ is the stress tensor denoted by:

$$
\overline{\bar{\sigma}}_{\mathrm{p}}=-\mathrm{p} \overline{\overline{\mathrm{I}}}+\mu\left[\nabla \overrightarrow{\mathrm{u}}+(\nabla \overrightarrow{\mathrm{u}})^{T}\right]
$$

where $\overline{\mathrm{I}}$ is the second order unit tensor.

For a spherical particle, its rotation does not influence the focusing position and the above three equations (Eq.(12)-(14)) can be used to approximate particle motion. As regards to a non-spherical particle, the rotational component does contribute to the focusing position.

The rotational velocity of the particle is given by [50]:

$$
I_{p} \frac{d \vec{\omega}_{p}}{d t}=\vec{T}_{n e t}
$$

where $I_{p}$ is the moment of inertial of the particle and $\vec{T}_{n e t}$ is the total torque acting on the particle given by 


$$
\vec{T}_{n e t}=\int\left(\vec{x}_{s}-\vec{x}_{p}\right) \times\left(\bar{\sigma}_{\mathrm{p}} \cdot \vec{n}\right) d S
$$

The position of the particle is determined by

$$
\vec{x}_{p}=\vec{x}_{p_{0}}+\int_{0}^{\mathrm{t}} \vec{U}_{p} \mathrm{dt}
$$

and

$$
\vec{\theta}_{p}=\vec{\theta}_{p_{0}}+\int_{0}^{\mathrm{t}} \vec{\omega}_{\mathrm{p}} \mathrm{dt}
$$

where $\vec{x}_{p_{0}}$ and $\vec{\theta}_{p_{0}}$ are, respectively, the initial location and orientation of the particle.

The initial conditions of the particle motion and the flow velocity are all set as zero, that is:

$$
\vec{U}_{p}=0, \vec{\omega}_{\mathrm{p}}=0, \vec{\theta}_{p_{0}}=0 \text { and } \vec{U}=0 \text { at } \mathrm{t}=0
$$

\section{Numerical simulation}

In this study, COMSOL MULTIPJHYSICS $4.3 \mathrm{~b}^{\circledR}$ was used to solve this 2D numerical model. To avoid the grid dependence, we compared the width of the focused stream with different numbers of elements and found that the width value difference is less than $0.1 \%$ when the numbers of elements is between 8,000 and 9,500. Therefore, 9,000 triangular elements were used in all simulations. Table 1 gives the parameter values used in the simulation.

\section{Experimental validation}

\subsection{Experimental set up}

\section{(1) Microchannel ant its fabrication}

The microchannel, as shown in Figure 1(a), was fabricated by bonding a PDMS (polydimethylsiloxane) layer with a glass substrate using the soft lithography method [51]. The width, height and length of the channel are $100 \mu \mathrm{m}, 50 \mu \mathrm{m}$ and $1 \mathrm{~cm}$ respectively. Considering the symmetry, only one metal plate was employed in the experimental studies to demonstrate the effect of the vortices on alternating the trajectories of the particles. The copper plate is $250 \mu \mathrm{m}$ long and $40 \mu \mathrm{m}$ thick and is embedded in one side wall of the microchannel. To embedding the metal plate along the channel wall, the metal plate was 
aligned with the negative photo-resist (SU-8 2025, MicroChem Co., Newton, MA) channel wall on a silicon master (4" N/PHOS, Montco Silicon Technology Inc., Spring City, PA) fabricated by the soft lithography method under a microscope(Ti-E, Nikon, Japan). Afterwards, a liquid PDMS layer (Sylgard 184, Dow Corning, USA) of 1 2mm thick was spin-coated onto the master and heated at $80^{\circ} \mathrm{C}$ in an oven for $1-2 \mathrm{~h}$. The PDMS layer, with the embedded metal plate along the wall, was then carefully peeled off from the master and bonded to a glass substrate $(25.66 \times 75.47 \times 1.07 \mathrm{~mm}$, CITOGLAS, China) using a plasma cleaner (HARRICK PLASMA). The fabrication procedure is shown in Figure 2.

\section{(2) Experimental procedures}

In this study, the trajectories of $2 \mu \mathrm{m}$ spherical polystyrene particles (Fluka, Shanghai, China) in pure water under different electrical fields $(\mathrm{E}=0,31,46$ and $62 \mathrm{~V} / \mathrm{cm})$ were experimentally investigated. The pure water was generated by a Millipore pure water system with a conductivity of about $5.5 \mu \mathrm{S} / \mathrm{m}$. In the case of $\mathrm{E}=0 \mathrm{~V} / \mathrm{cm}$, a small pressure difference of approximate $0.05 \mathrm{~Pa}$ (by a liquid level difference between the inlet well and the outlet well) was applied across the channel in order to transport the particles. To measure particle trajectories under non-zero electrical fields, the liquid levels in the inlet and outlet wells were carefully balanced by adding a certain amount of pure water in the wells with a digital micro-pipette. Then Pt electrodes were inserted in the wells and an electrical field was applied across the channel. In the meantime, the particle motion was recorded by the CCD (Charge Coupled Device) camera (DS-Qi1Mc, Nikon) of an inverted optical microscope imaging system (Ti-E, Nikon). The camera was operated in a video mode at a frame rate of 11.4 frames per second. The reading error in determining the cell position is about \pm 2 pixels which correspond to actual dimension of $\pm 5.4 \mu \mathrm{m}$.

To minimize the influence of static hydraulic pressure on particle movement, relatively larger wells were used to minimize the back pressure driven flow generated by the electroosmotic flow. Also, only the particles moving close to the channel wall before the metal plate were followed and their trajectories were recorded in order to examine the electrical field effect on the particle trajectory.

\subsection{Experimental results and comparison}


Figure 3(a) shows the experimentally-obtained trajectories of $2 \mu \mathrm{m}$ particles under different electrical fields. The trajectories were obtained by superposing a series of consecutive images of the moving particles. As explained in the previous section (Working Principle), there are two vortices over the metal plate in the induced charge electroosmotic flow. Although the two vortices cannot be seen in Figure 3(a), it is clear that the particles firstly pass along the first half of the metal plate (under the $1^{\text {st }}$ vortex as illustrated in Figure 1(b)). When approaching the length center of the metal plate, the particles were directed towards the channel center and away from the metal plate, resulting from the action of the $2^{\text {nd }}$ vortex. The particles move much farther away from the second half of the metal plate under larger electrical field, meaning that the particle's trajectory can be controlled by controlling the applied electrical field. As a comparison, the simulated particle trajectories are also shown in Figure 3(b). As can be seen from Figure 3, the experimental results agree reasonably well with the numerical simulations.

In the experimental studies, the metal plates were manually placed on the side wall of the microchannel under a microscope. For focusing particles with two metal plates, the focusing position will be affected by the asymmetrical vortices, if the two metal plates are not exactly aligned symmetrically on the two facing channel walls. In order to avoid such uncertainty, in the comparison shown in Figure 3, only one metal plate was used to validate the working principle and demonstrate the focusing effect of this novel method. The focusing effects with two plates will be numerically investigated and discussed as below.

\section{Focusing effects by numerical predictions}

Convinced by the above results of controlling particle trajectories with one metal plate under different electrical fields, the width and length of the focused particle stream with two metal plates under different conditions were further investigated by numerical simulations and using the mathematical model described above. For the simulations, the composition of metal plate surface is considered to be not changed with time and no chemical reactions happen. It should be noted, however, the dielectric constant will be greatly decreased and thus decrease the strength of the vortices, if the metal can be oxidized by the solution. 


\subsection{Dependence of the focused width and length on electrical field}

Figure 4 shows the flow field (streamlines) of the particle solution in the region of the metal plates $\left(\mathrm{L}_{\mathrm{m}}=50 \mu \mathrm{m}\right)$ under an electrical field of $180 \mathrm{~V} / \mathrm{cm}$. It's clear that the stream of the particle solution will be narrowed between the two downstream vortices. The size of the particle solution stream between the two vortices depends on the size of the vortices which is affected by the applied electrical field.

Generally, the size and structure of the channel are not changed in practice. Different focusing effects, especially the focused width and length, may be required for different purposes. Thus, it is greatly desired to know the focused width and length under different applied electrical fields for a channel with constant width. Figure 5 shows the dependence of the width ratio and length ratio of the focused particle stream on the applied electrical field. As can be seen from Figure 5(a), the focused width ratio decreases with the increase in the electrical field. This means that the particle stream becomes thin with the increase in the applied electrical field. This can be understood by the fact that increasing the applied electrical field will increase the induced zeta potential (induced surface charge), and hence the size of the vortices, which will leave a smaller gap for the particle solution to pass. In other words, the passage width for the particle stream can be easily adjusted by controlling the applied electrical field. With respect to the length of the focused particle stream, it is determined by the length of the two downstream vortices which in turn are determined both by the length of the metal plate and the applied electrical field. As is shown in Figure 5(b), the length ratio slightly increases with the increase in the applied electrical field. As will be discussed in Section 6.2, the length of the focused stream is much influenced by that of the metal plate. With the relationship shown in Figure 4, one can easily obtain different focusing effects by choosing different electrical fields.

\subsection{Dependence of the focused width and length on metal plate length}

Varying the length of the metal plate is another important approach to acquiring different focusing effect. Figure 6 shows the relationship between the width ratio and length ratio of the focused particle stream on the length of the metal plate under an electrical field of 200 
$\mathrm{V} / \mathrm{cm}$. As seen from Figure 6, the increase in the length of the metal plate will narrow the width of the focused particle stream, and increase linearly the length of the focused stream (with a constant length ratio). This is due to larger induced surface potentials (named as idealized surface potential) with longer metal plate under the same applied electrical field, as is clearly shown in Figure 7. From Figure 7, it's clear that the magnitude of the induced potentials at the ends of the metal plate become larger for a longer metal plate. The larger induced potentials will result in stronger vortices (larger in size) which will make the focused sample solution narrower and longer. Comparing with the way of varying the applied electrical field, this approach seems to be more attractive in terms of avoiding the damaging effects on cells caused by applying large electrical field.

\subsection{Dependence of the focused width and length ratio on channel width}

As mentioned above, the flow passage between the two downstream vortices are determined by the occupied space of the vortices in the channel. However, the vortices are constrained by the channel which will in turn influence the size of the vortices. In other words, the distance between the two metal plates can influence the focusing effects. Figure 8 shows the dependence of the focused width and length ratios on the width of the channel. It's clear that wider focused width and longer focused length were resulted for a wider channel under the same electrical field and the metal plates. The electroosmotic flow velocities at the PDMS channel walls and at the metal plates remain the same, independent of the increase in channel width. For a wider channel, however, the interaction between the two downstream vortices will be weaker due to the weaker constrain imposed by the channels. As a result, the focused width is wider and the focused length becomes longer.

\section{Other discussions}

\section{(1) Particle selectivity by size}

While only particle focusing effects is presented for this method in this study, the separation of particles based on size may also be achieved and can be understood as bellow: The gap 
between the two downstream vortices, which is the passage of the solution or particles, can be flexibly controlled by adjusting the applied electrical field, based on the established relationship between the focused width and the applied electrical field. For particles larger than the gap size, they will be prevented from passing through gap, thus achieving particle separation based on size difference.

\section{(2) Ion concentration effects}

The induce zeta potential over a metal plate (as an ideal, perfectly polarizable material) is independent of the ion concentration of the solution. Therefore, as indicated by Eq. (1), the electroosmotic flow at the metal plate-solution interface (named as EOF 1) is independent of the ion concentration of the solution. As explained above, EOF1 has vortices.

The zeta potential and the thickness of electric double layer at the interface between a dielectric material such as PDMS and the aqueous solution depend on the ion concentration of the solution. The higher the ionic concentration, the lower the zeta potential and hence the weaker the electroosmotic flow. In the system studied in this paper, the laminar electroosmotic flow from the upstream PDMS channel (named as EOF 2) strikes the downstream vortex flow (EOF 1) and thus influence the formation and the size of the vortices. A stronger EOF2 will result in smaller vortices over the metal plates. Increasing the ion concentration (of course the conductivity) of the solution will reduce the zeta potential and the EOF from the upstream PDMS channel, and hence facilitate the formation of the vortices near the focusing region, and can improve the focusing effect.

\section{(3) Electrical field related side effects}

For this particle focusing method, the side effects caused by applying an electrical field, such as cell damage by electrical field and Joule heating, have to be considered. In our study, the highest field intensities at metal plate edges are 117, 173 and $233 \mathrm{~V} / \mathrm{cm}$ under the applied electric field of $31 \mathrm{~V} / \mathrm{cm}, 46 \mathrm{~V} / \mathrm{cm}$ and $62 \mathrm{~V} / \mathrm{cm}$ respectively. In comparison to most insulator based DC-dielectrophoretic platforms, the strength of the electric field is significantly small and therefore the damage to the cell caused by electrical field can be avoided. 
The Joule heat, generated by the electrical field, is proportional to the liquid electrical conductivity (the solution's electrical concentration). According to the reference paper [52], under an electric field of $600 \mathrm{~V} / \mathrm{cm}$, the Joule heating can be safely neglected for dilute solutions (e.g., $\mathrm{C}=10^{-4} \mathrm{M}$ ). In our study, the highest electrical field applied across the channel is $62 \mathrm{~V} / \mathrm{cm}$ and the solution is pure water generated by a Millipore pure water system. The conductivity of the pure water is about $5.5 \mu \mathrm{S} / \mathrm{m}$. Therefore, the influence of Joule heat on focusing can be neglected.

\section{Conclusion}

A novel sheathless particle focusing method by induced charge electrokinetic flow (ICEK) in a microchannel was developed in this paper. Particle focusing can be achieved by placing two metal plates on opposite channel walls. Both numerical and experimental studies show that the particles firstly will be transported through the gaps between the upstream vortices and the channel walls. Afterwards, the particles will be focused to pass through the passage between the two downstream vortices. The width of the focused particle stream decreases with the increase in the applied electrical field and the metal plate length. The length of the focused stream increases with the increase in the applied electrical field and the metal plate length. In short, the size of the focused sample solution can be easily adjusted by controlling the applied electrical field and the size of the metal plate. Comparing with other focusing techniques, the method presented in this paper is simple and efficient without using sheath fluids or other off-chip devices.

\section{Acknowledgement}

The authors wish to thank the financial support of the Fundamental Research Funds for the Central Universities (3132014336), Liaoning Science Foundation (2014025020) and Liaoning Excellent Talent supporting Plan (LJQ2014050), Dalian Science and Technology Plan (2014E12SF068) from China to Yongxin Song, National Science Foundation program of 
China (51479020) to Xinxiang Pan, the Natural Sciences and Engineering Research Council of Canada through a research grant to D. Li and the financial support from the University 111 project of China under Grant No. B08046 is greatly appreciated. 


\section{Reference}

[1] Xuan, X., Zhu, J., Church, C., Microfluid. Nanofluid. 2010, 9, 1-16.

[2] Akagi, J., Kordon ,M., Zhao, H., Matuszek, A., Dobrucki, J., Errington, R., Smith, P. J., Takeda, K., Darzynkiewicz, Z., Wlodkowic, D., Cytom. Part A. 2013, 83, 227-234.

[3] Shi, J., Mao, X., Ahmed, D., Colletti, A., Huang, T. J., Lab Chip 2008, 8, 221-223.

[4] Xuan, X., Li, D., Electrophoresis 2005, 26, 3552-3560.

[5] Jacobson, S. C., Ramsey, J. M., Anal. Chem.1997, 69, 3212-3217.

[6] Schrum, D. P., Culbertson, C. T., Jacobson, S. C., Ramsey, J. M., Anal. Chem.1999, 71, 4173-4177.

[7] Fu, L. M., Yang, R. J., Lee, G. B., Anal. Chem.2003, 75, 1905-1910.

[8] Fu, L. M., Yang, R. J., Lin, C. H., Pan, Y. J., Lee, G. B., Anal.Chim.Acta. 2004, 507, 163-169.

[9] Yang, R. J., Chang, C. C., Huang, S. B., Lee, G. B., J. Micromech. Microeng.2005, 15, 2141.

[10] Xuan, X., Li, D., Electrophoresis 2005, 26, 3552-3560.

[11] Godin, J., Chen, C. H., Cho, S. H., Qiao, W., Tsai, F., Lo, Y. H., J.Biophotonic. 2008, 1, 355-376.

[12] Ateya, D. A., Erickson, J. S., Howell Jr, P. B., Hilliard, L. R., Golden, J. P., Ligler, F. S., Anal. Bioanal.Chem.2008, 391, 1485-1498.

[13] Daniele, M. A., Boyd, D. A., Mott, D. R., Ligler, F. S., Biosens. Bioelectron. 2015, 67, 25-34.

[14] Liu, C., Stakenborg, T., Peeters, S., Lagae, L., J. Appl. Phys. 2009, 105, 102014.

[15] Zhao, Y., Fujimoto, B. S., Jeffries, G. D., Schiro, P. G., Chiu, D. T., Opt. Express. 2007, $15,6167-6176$.

[16] Pohl, H. A., J. Appl. Phys. 1951, 22, 869-871.

[17] Lin, C. H., Lee, G. B., Fu, L. M., Hwey, B. H., J. Microelectromech Syst. 2004, 13, 923-932.

[18] Yu, C., Vykoukal, J., Vykoukal, D. M., Schwartz, J.A., Shi, L., Gascoyne, PR. C., J. Microelectromech Syst. 2005, 14, 480-487. 
[19] Holmes, D., Morgan, H., Green, N.G., Biosens. Bioelectron.2006, 21, 1621-1630.

[20] Cheng, I. F., Chang, H. C., Hou, D., Chang, H. C., Biomicrofluidics 2007, 1, 1-15.

[21] Lapizco-Encinas, B.H., Rito-Palmomares, M., Electrophoresis 2007, 28, 45214538 .

[22] Demierre, N., Braschler, T., Muller, R., Sens. Actuat. B Chem. 2008, 132, 388-396.

[23] Braschler, T., Demierre, N., Nascimento, E., Silva, T., Oliva, A.G., Renaud, P., Lab Chip 2008, 8, 280-286.

[24] Wang, L., Lu, J., Marchenko, S. A., Monuki, E. S., Flanagan, L. A., Lee, A. P., Electrophoresis 2009, 30, 1-10.

[25] Cheri, M. S., Latifi, H., Khashei, H., Seresht, M. J., Electrophoresis 2014, 35, 3523-3532.

[26] Chu, H., Doh, I., Cho, Y. H., Lab Chip 2009, 9, 686-691.

[27] Li, M., Li, S., Cao, W., Li, W., Wen, W., Alici, G., Microfluid. Nanofluid. 2013, 14, $527-539$.

[28] Demierre, N., Braschler, T., Muller, R., Renaud, P., Sens. Actuat. B Chem. 2008, 132, 388-396.

[29] Morgan, H., Holmes, D., Green, N. G., Nanobiotechnology 2003, 150, 76-81.

[30] Qian, C., Huang, H., Chen, L., Li, X., Ge, Z., Chen, T., Yang, Z., Sun, L., Int. J. Mol. Sci. 2014, 15, 18281-18309.

[31] Jubery, T. Z., Srivastava, S. K., Dutta, P., Electrophoresis 2014, 35, 691-713.

[32] Shi, J., Yazdi, S., Lin, S. C. S., Ding, X., Chiang, I. K., Sharp, K., Huang, T. J., Lab Chip 2011, 11, 2319-2324.

[33] Goddard, G. R., Sanders, C. K., Martin, J. C., Kaduchak, G., Graves, S. W., Anal.Chem. 2007, 79, 8740-8746.

[34] Suthanthiraraj, P. P. A., Piyasena, M. E., Woods, T. A., Naivar, M. A., López, G. P., Graves, S. W., Methods. 2012, 57, 259-271.

[35] Grenvall, C., Antfolk, C., Bisgaard, C. Z., Laurell, T., Lab Chip 2014, 14, 4629-4637.

[36] Antfolk, M., Muller, P. B., Augustsson, P., Bruus, H., Laurell, T., Lab Chip 2014, 14, 2791-2799.

[37] Jakobsson, O., Grenvall, C., Nordin, M., Evander, M., Laurell, T., Lab Chip 2014, 14, 
1943-1950.

[38] Jiang, H., Weng, X., Li, D., Biomicrofluidics 2014, 8, 054120.

[39] Di Carlo, D., Lab Chip 2009, 9, 3038-3046.

[40] Amini, H., Lee, W., Di Carlo, D., Lab Chip 2014, 14, 2739-2761.

[41] Martel, J. M., Toner, M., Annu.Rev. Biomed. Eng. 2014, 16, 371-396.

[42] Liu, C., Hu, G., Jiang, X., Sun, J., Lab Chip 2015,15, 1168-1177.

[43] Hood, K., Sungyon, Lee., Roper, M., J. Fluid. Mechanics 2015, 452, 79.

[44] Chen, H., Zhang, Y., Mezic Igor, Meinhart Carl and Linda Petzold, 2003 ASME International Mechanical Engineering Congress and Exposition, 2003.

[45] Zhe, M. Wu., Dong, Q. Li., Electrochim. Acta. 2008, 53, 5827-5835.

[46] R, J. Hunter., Academic. Press. New York, 1981, 364.

[47] D, Li., Elsevier. Academic. Press. 2004.

[48] Ye, C., Li, D., J. Colloid Interface Sci. 2004, 272, 480-488.

[49] Wu, Z., Gao, Y., Li, D., Electrophoresis 2009, 30, 773-781.

[50] Ai, Y., Joo, S. W., Jiang, Y., Electrophoresis 2009, 30, 2499-2506.

[51] Xia, Y. N., Whitesides, G. M., Ann. Rev. Mater. Sci. 1998, 28, 153-184.

[52] Tang, G., Yan, D., Yang, C., Gong, H., Chai, C., Lam, Y., Sens. Actuat. A Phys. 2007, 139, 221-232. 


\section{Table 1}

Parameters used in the numerical simulations

\begin{tabular}{ccc}
\hline Parameters & Values & Units \\
\hline$\varepsilon_{f}$ & 80 & - \\
$\varepsilon_{0}$ & $8.85 \times 10^{-12}$ & $\mathrm{~F} / \mathrm{m}$ \\
$\mu$ & $1 \times 10^{-3}$ & $\mathrm{Pas}$ \\
$\rho$ & $1 \times 10^{3}$ & $\mathrm{~kg} / \mathrm{m}^{3}$ \\
$\zeta_{w}$ & -110 & $\mathrm{mV}$ \\
$\zeta_{p}$ & -20 & $\mathrm{mV}$ \\
\hline
\end{tabular}




\section{Figure Legends}

Figure 1 A schematic diagram of ICEK particle focusing, (a) The induced charge and electrical double layers in a microchannel with two embedded metal plates, (b) Illustration of the vortices and the focused particle trajectory.

Figure 2 A schematic diagram of the microchip fabrication process.

Figure 3 Trajectories of $2 \mu \mathrm{m}$ particles in a microchannel with only one metal plate on one side channel wall under different electrical fields, (a) experimental results, (b) numerical simulation results.

Figure 4 Flow field in the region of the metal plates $\left(\mathrm{L}_{\mathrm{m}}=50 \mu \mathrm{m}\right)$ under an electrical field of $180 \mathrm{~V} / \mathrm{cm} . \mathrm{W}$ is the width of the microchannel; $\mathrm{L}$ is the length of the metal plate; $W_{\mathrm{f}}$ and $L_{\mathrm{f}}$ are the focused particle stream, respectively.

Figure 5 Dependence of the focused width ratio (a) and the focused length ratio (b) on electrical field $\left(\mathrm{L}_{\mathrm{m}}=100 \mu \mathrm{m}, \mathrm{W}=20 \mu \mathrm{m}\right)$

Figure 6 Dependence of the focused width ratio (a) and the focused length ratio (b) on the length of the metal plates $(\mathrm{E}=200 \mathrm{~V} / \mathrm{cm}, \mathrm{W}=20 \mu \mathrm{m})$

Figure 7 Dependence of the idealized surface potential on the length of the metal plates (E $=200 \mathrm{~V} / \mathrm{cm})$

Figure 8 Dependence of the focused width and length ratios on the width of the channel. 


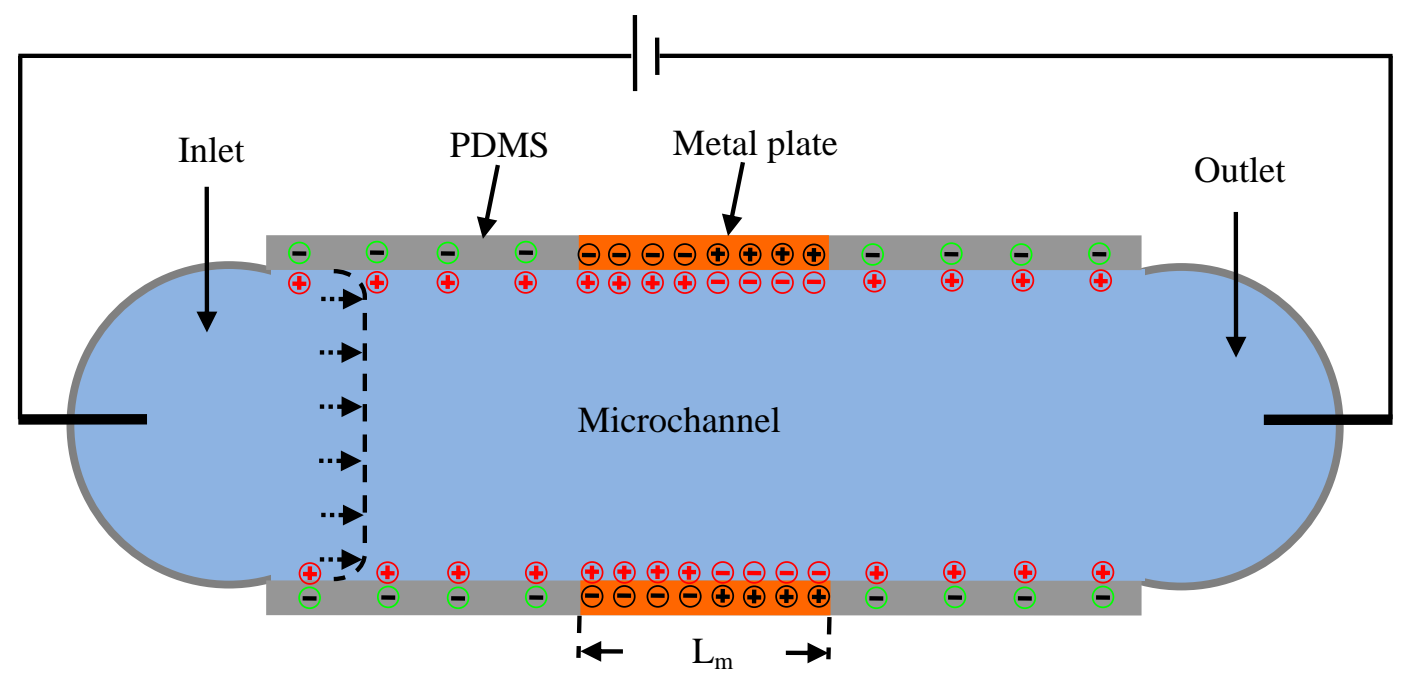

(a)

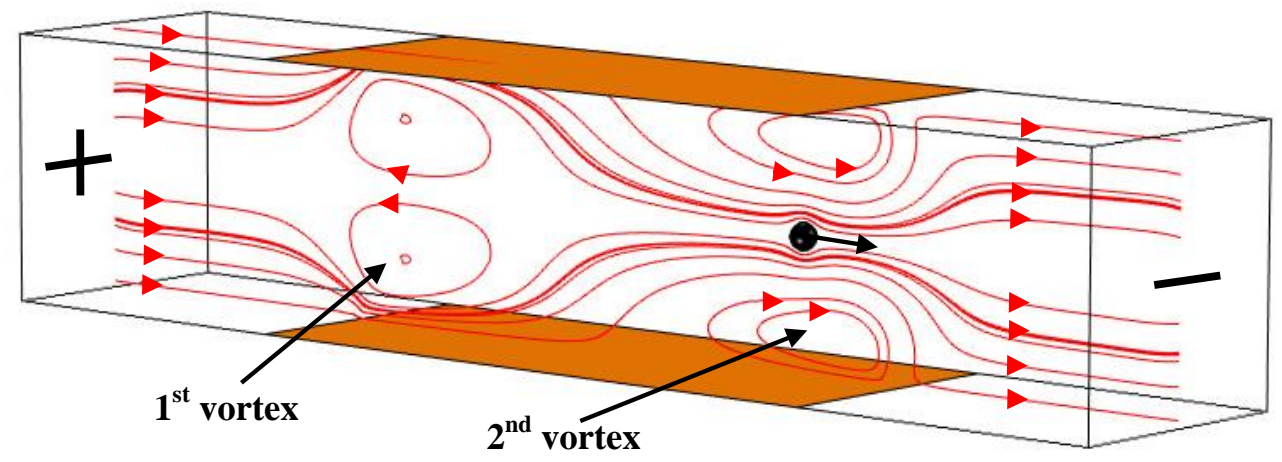

(b)

Figure 1 


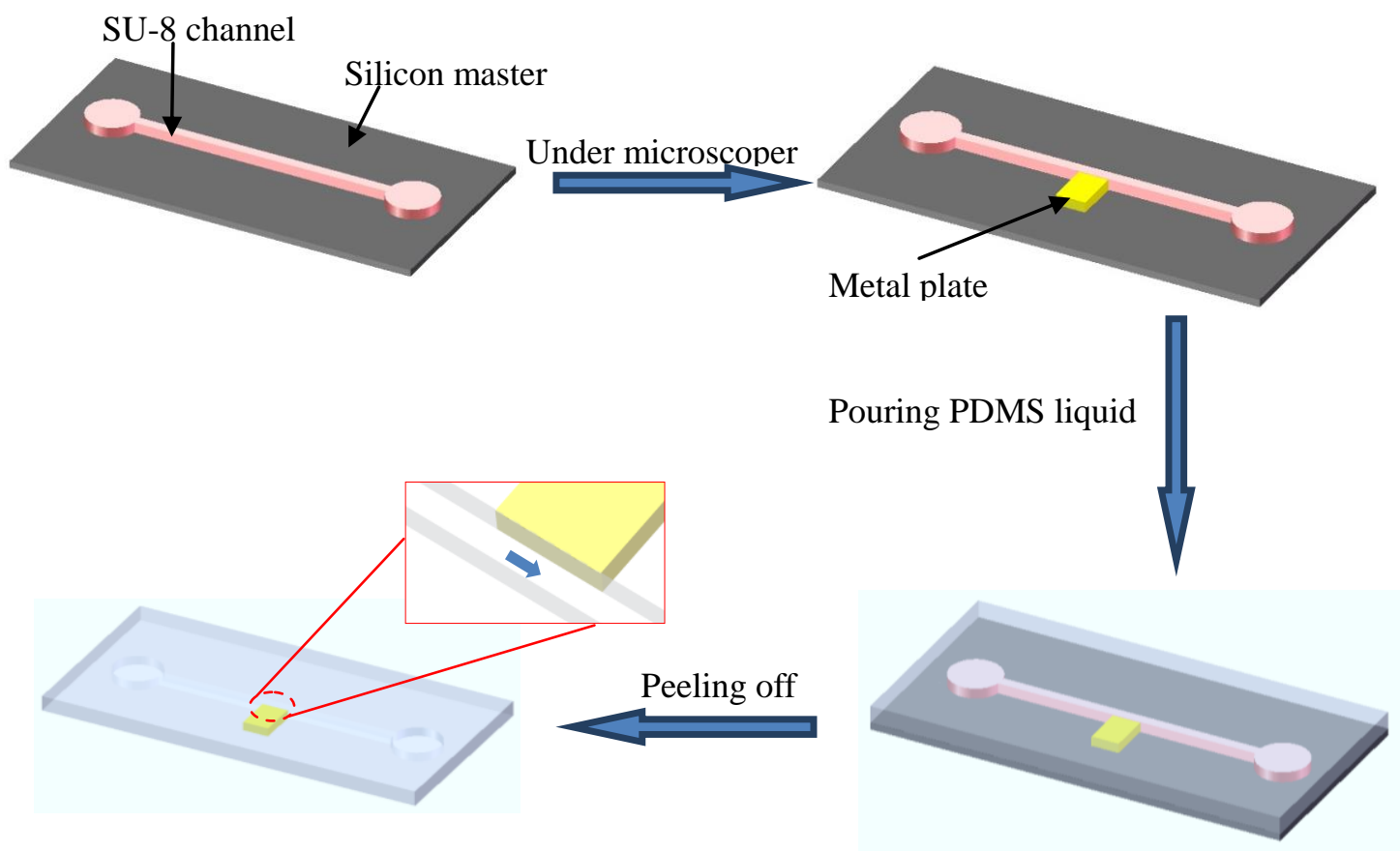

Figure 2 


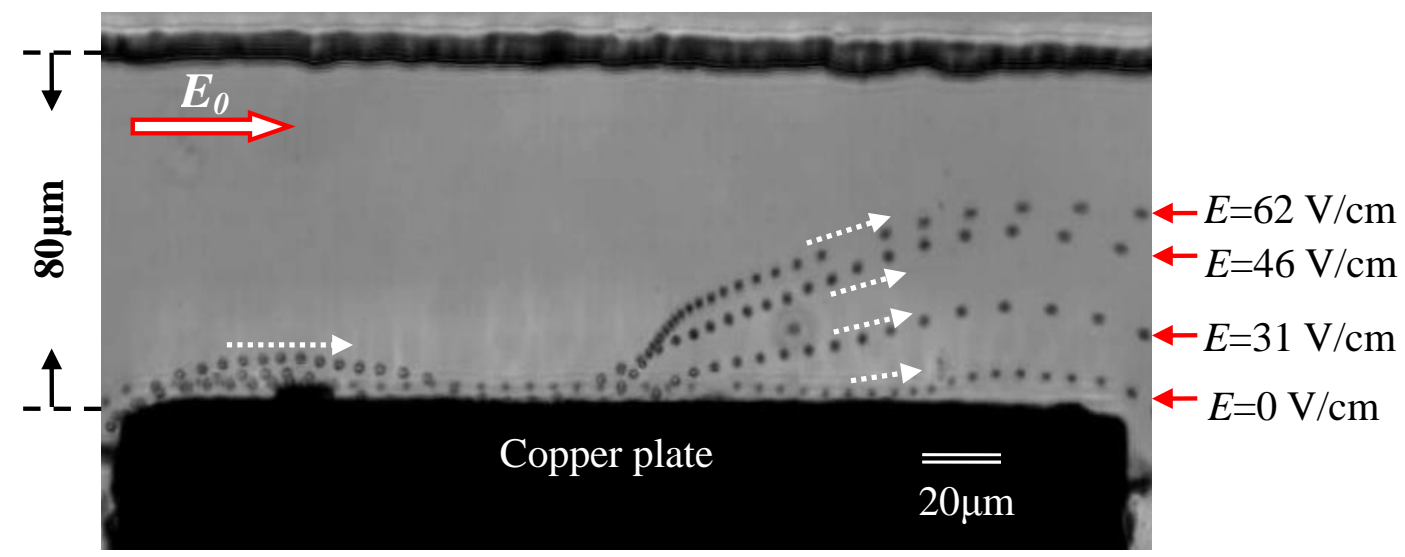

(a)

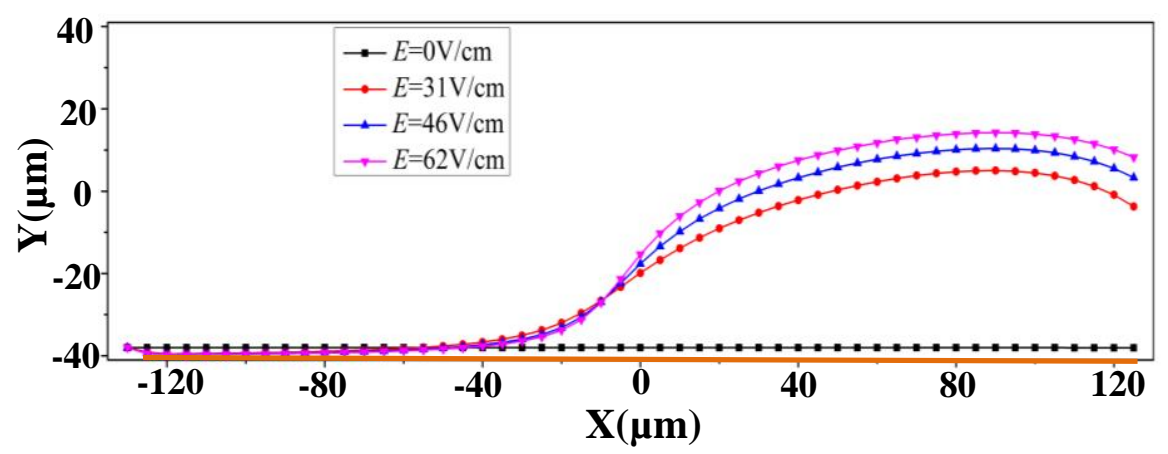

(b)

Figure 3 


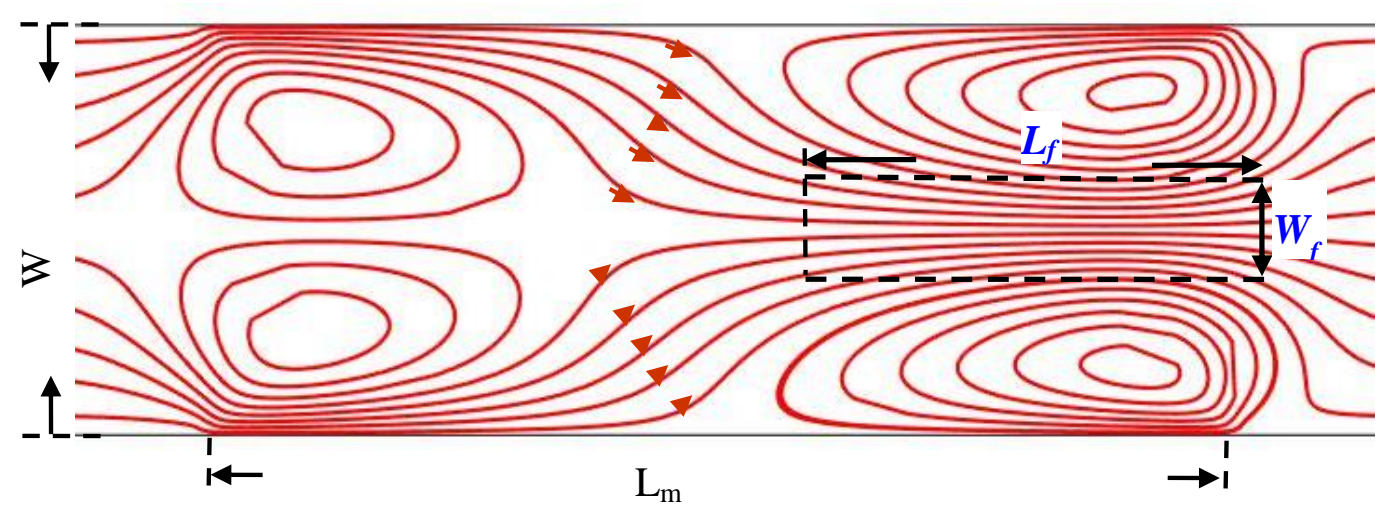

Figure 4 


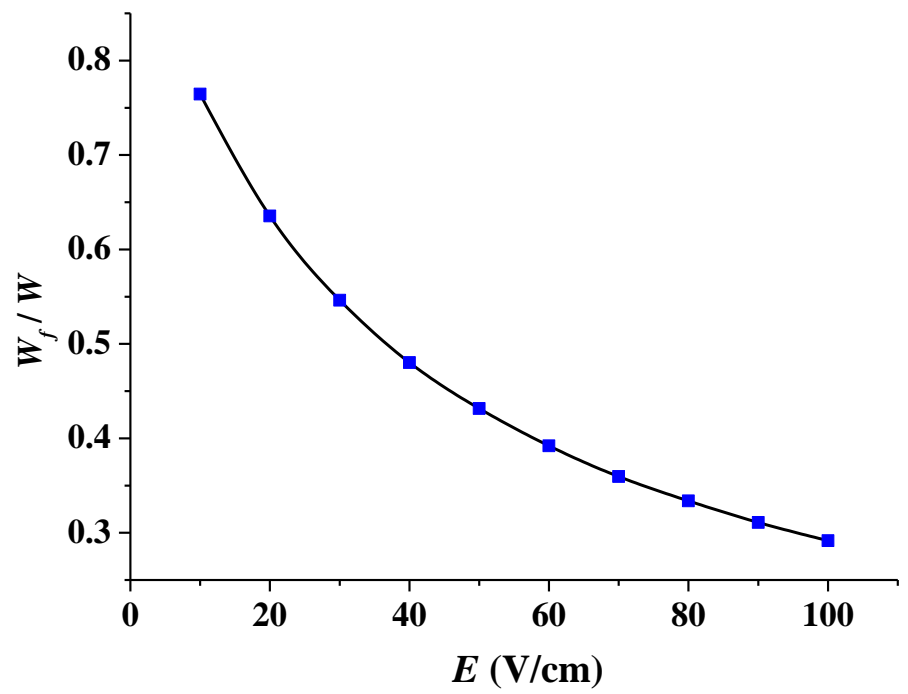

(a)

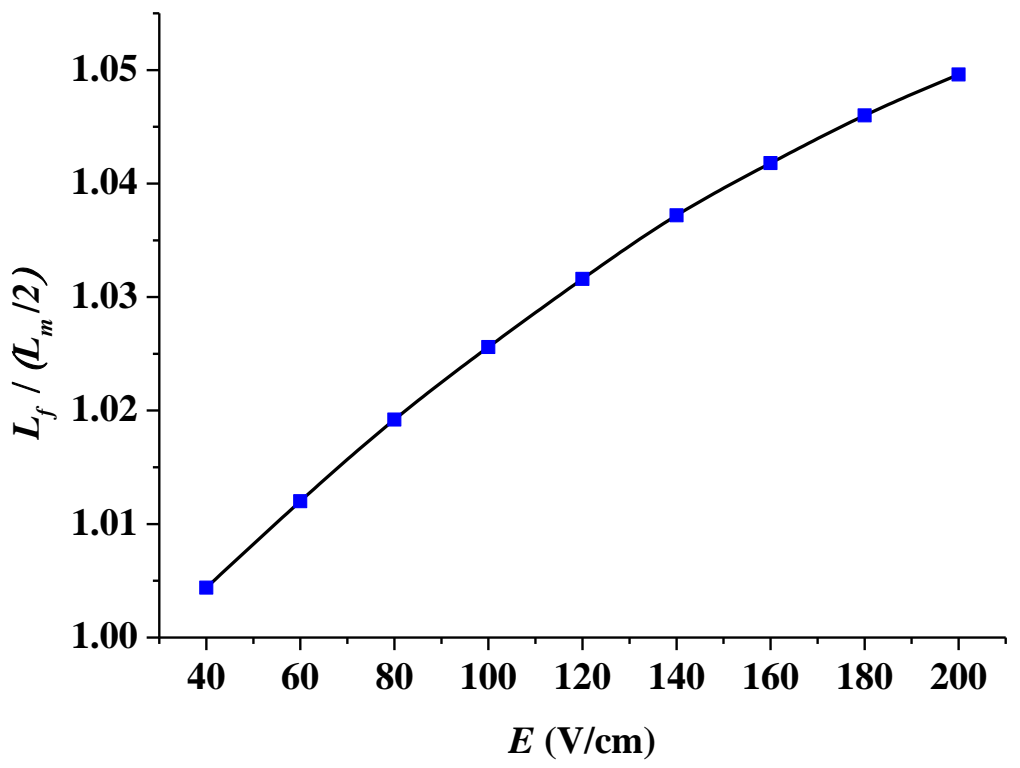

(b)

Figure 5 

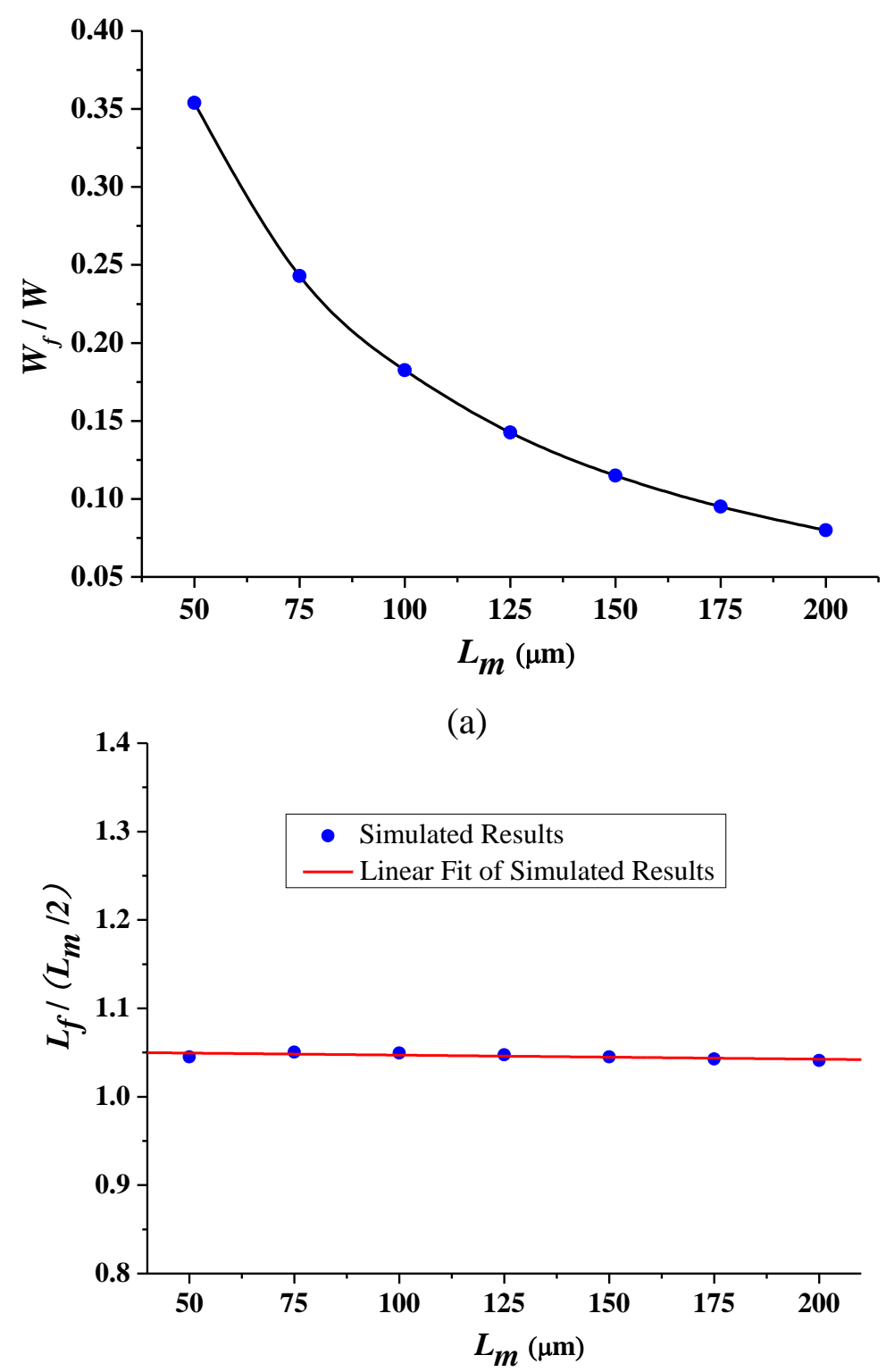

(b)

Figure 6 


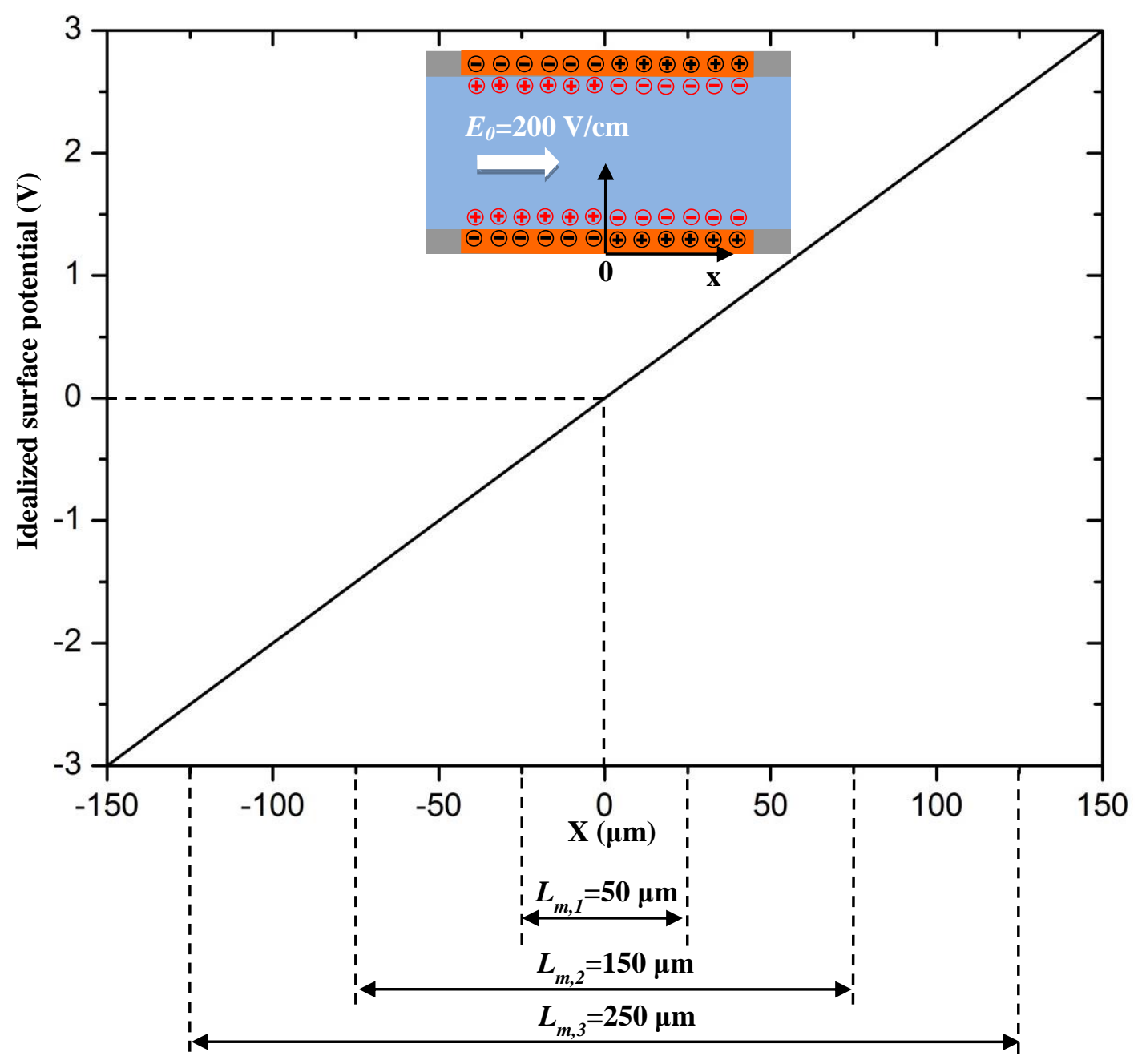

Figure 7 


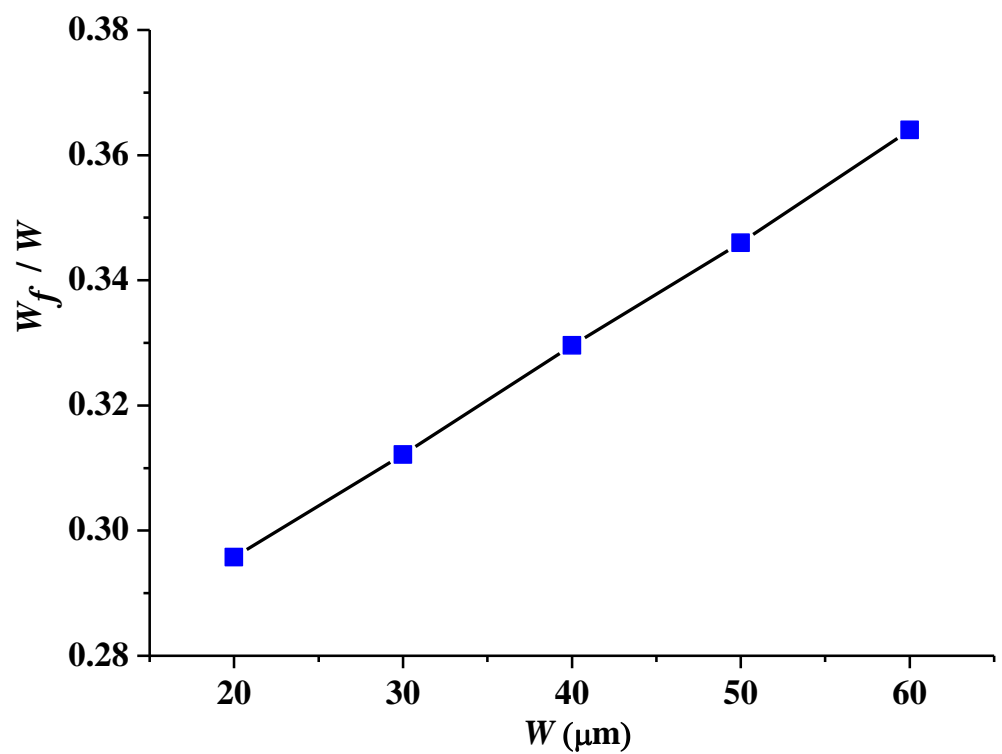

(a)

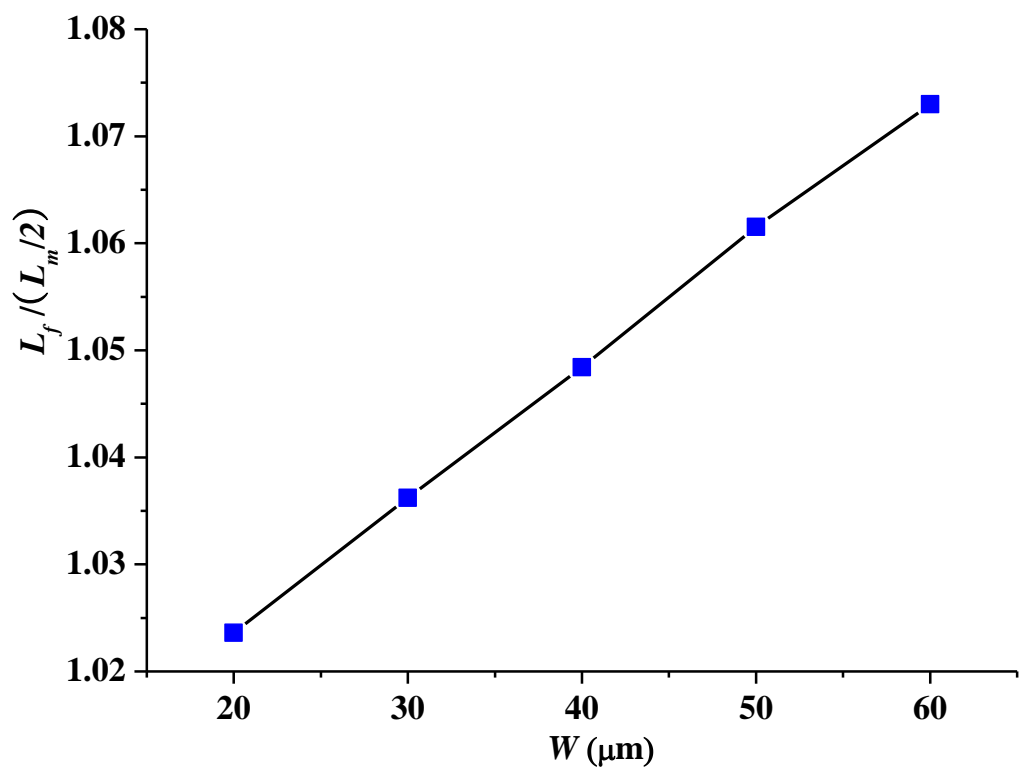

(b)

Figure 8 